\title{
ARTICLE OPEN Resolving the phylogenetic origin of glioblastoma via multifocal genomic analysis of pre-treatment and treatment- resistant autopsy specimens
}

Priscilla K. Brastianos ${ }^{1,2,3,4,5}$, Naema Nayyar ${ }^{2,4,5}$, Daniel Rosebrock ${ }^{2}$, Ignaty Leshchiner (iD ${ }^{2}$, Corey M. Gill ${ }^{4,5}$, Dimitri Livitz (iD ${ }^{2}$, Mia S. Bertalan ${ }^{4,5}$, Megan D'Andrea ${ }^{4,5}$, Kaitlin Hoang ${ }^{4,5}$, Elisa Aquilanti ${ }^{1,2,3,4,5}$, Ugonma N. Chukwueke ${ }^{4,5}$, Andrew Kaneb ${ }^{4,5}$, Andrew Chi ${ }^{6}$, Scott Plotkin ${ }^{1,3,4,5}$, Elizabeth R. Gerstner ${ }^{1,3,4,5}$, Mathew P. Frosch ${ }^{3,7}$, Mario L. Suva $\mathbb{D}^{3,7}$, Daniel P. Cahill ${ }^{3,5,8}$, Gad Getz $\mathbb{1 D}^{2,3,5,7}$ and Tracy T. Batchelor ${ }^{1,3,4,5}$

Glioblastomas are malignant neoplasms composed of diverse cell populations. This intratumoral diversity has an underlying architecture, with a hierarchical relationship through clonal evolution from a common ancestor. Therapies are limited by emergence of resistant subclones from this phylogenetic reservoir. To characterize this clonal ancestral origin of recurrent tumors, we determined phylogenetic relationships using whole exome sequencing of pre-treatment IDH1/2 wild-type glioblastoma specimens, matched to post-treatment autopsy samples $(n=9)$ and metastatic extracranial post-treatment autopsy samples $(n=3)$. We identified "truncal" genetic events common to the evolutionary ancestry of the initial specimen and later recurrences, thereby inferring the identity of the precursor cell population. Mutations were identified in a subset of cases in known glioblastoma genes such as NF1 $(n=3), \operatorname{TP53}(n=4)$ and $\operatorname{EGFR}(n=5)$. However, by phylogenetic analysis, there were no protein-coding mutations as recurrent truncal events across the majority of cases. In contrast, whole copy-loss of chromosome 10 (12 of 12 cases), copy-loss of chromosome 9p21 (11 of 12 cases) and copy-gain in chromosome 7 (10 of 12 cases) were identified as shared events in the majority of cases. Strikingly, mutations in the TERT promoter were also identified as shared events in all evaluated pairs (9 of 9). Thus, we define four truncal non-coding genomic alterations that represent early genomic events in gliomagenesis, that identify the persistent cellular reservoir from which glioblastoma recurrences emerge. Therapies to target these key early genomic events are needed. These findings offer an evolutionary explanation for why precision therapies that target protein-coding mutations lack efficacy in GBM.

npj Precision Oncology (2017)1:33 ; doi:10.1038/s41698-017-0035-9

\section{INTRODUCTION}

Glioblastoma (GBM) is the most common primary malignant brain tumor, with a poor prognosis. Therapies (including therapies that target specific alterations) that have shown efficacy in other cancers have failed in GBM. In the past 3 decades, only a single cytotoxic chemotherapeutic agent, temozolomide (TMZ), has been approved and widely used for GBM and this drug only modestly extends survival. Although the genomics of GBM at diagnosis have been extensively characterized ${ }^{1-3}$, the existence and identity of genomic drivers leading to GBM progression and recurrence remain elusive.

Starting from a normal cell, cancers evolve via multiple rounds of mutation, selection, and expansion. ${ }^{4,5}$ Continued elaboration of this phylogenetic process within the growing cancer-cell population results in branched genetic variegation, ${ }^{6}$ whereby multiple cancer subclones relate to each other in a phylogenetic tree-like fashion. ${ }^{7}$ Consequently, cancer biospecimens are substantially heterogeneous both across different anatomical regions ${ }^{8-11}$ and within single cancer biopsies. ${ }^{1-15}$

GBM, when compared to many other cancers ${ }^{16}$, is a genetically heterogeneous disease. Multiregional sampling of GBM at a single timepoint commonly demonstrates significant intratumoral heterogeneity. ${ }^{17-19}$ Studies of matched pre-treatment and recurrent GBM after failure of therapy remain limited ${ }^{20-22}$ especially at the extremes of disease, in large part due to the logistical challenges associated with obtaining tissue at recurrence or the time of death. The ongoing evolutionary processes leading to GBM recurrence, and ultimately death of the patient, remain largely uncharacterized.

Our objectives were to comprehensively characterize intratumoral heterogeneity and evolutionary patterns in GBM over the entire course of clinical care, from initial diagnosis to time of death. We initiated a GBM autopsy program at Massachusetts General Hospital, which offers us the ability to compare the

\footnotetext{
${ }^{1}$ Division of Hematology/Oncology, Massachusetts General Hospital, Boston, Massachusetts, USA; ${ }^{2}$ Broad Institute of MIT and Harvard, Boston, Massachusetts, USA; ${ }^{3}$ Harvard Medical School, Boston, Massachusetts, USA; ${ }^{4}$ Division of Neuro-Oncology, Massachusetts General Hospital, Boston, Massachusetts, USA; ${ }^{5}$ Cancer Center, Massachusetts General Hospital, Boston, Massachusetts, USA; ${ }^{6}$ Laura and Isaac Perlmutter Cancer Center, NYU Langone Medical Center, New York, NY, USA; ${ }^{7}$ Department of Pathology, Massachusetts General Hospital, Boston, Massachusetts, USA and ${ }^{8}$ Department of Neurosurgery, Massachusetts General Hospital, Boston, Massachusetts, USA Correspondence: Priscilla K. Brastianos (pbrastianos@mgh.harvard.edu) or Tracy T. Batchelor (TBATCHELOR@mgh.harvard.edu) Priscilla K. Brastianos, Naema Nayyar, Daniel Rosebrock, Ignaty Leshchiner contributed equally to this work. Daniel P. Cahill, Gad Getz and Tracy T. Batchelor jointly supervised this work.
} 
evolution of genetic changes at diagnosis, during treatment, and at the time of tumor progression and death. This served as the basis for a phylogenetic analysis of GBM throughout the disease course, as described herein.

\section{RESULTS}

We identified GBM patients from our autopsy tissue bank and acquired pre-treatment tissue from diagnosis and matched posttreatment autopsy tissue. We performed whole exome sequencing of 12 GBM cases for which we had tumor tissue separated by time $(n=10)$ and space $(n=2)$. Clinical characteristics of the 12patient case series are shown in Table 1. We also used a PCR-based assay followed by next generation sequencing to evaluate the presence and cancer cell fraction of the TERT promoter mutation.

We sequenced to high depth (mean target coverage $81 \mathrm{X}$ for the exome, 21,459X for the TERT promoter Fluidigm assay). The mean non-synonymous mutation rate in the post-treatment autopsy samples $(n=12)$ was 1.78 mutations/Mb (range 1.04 to 2.63 ) and the mean non-synonymous rate in the pre-treatment samples $(n=$ 9) was 1.19 mutations/Mb (range 0.81 to 1.50 ), consistent with prior reported mutation rates in $\mathrm{GBM}^{2}{ }^{2}$ There were no samples that detectably exhibited a 'hypermutator phenotype', as has been reported in a subset of $\mathrm{GBMs}^{23}$.

The most frequent point mutations were TERT promoter mutations, present in all patient cases where a Fluidigm assay was available $(n=11 / 11)$. For one case (GS-05), targeted sequencing data for the TERT promoter region was unavailable. Additionally, mutations that were previously reported in GBM were detected at a lower frequency compared to TERT alterations across our cohort, including mutations in NF1 ( $n=3$ patients, $25 \%$ cases), EGFR ( $n=5$, $41.7 \%$ cases), TP53 ( $n=4,33.3 \%), R B 1(n=1,8.3 \%), \operatorname{TSC} 1$ ( $n=1,8.3 \%)$. The most frequent copy number alterations were loss of chromosome 10 and 9p21.3, as well as broad chromosome 7 gain, distinct from focal EGFR amplification (Fig. 1a).

We applied previously described computational methods ${ }^{12,24-27}$ to address tumor heterogeneity and infer the evolutionary relationship between the matched, sequenced tissue samples from each patient. For each matched pre-treatment and posttreatment autopsy sample, we integrated copy-number alterations and somatic point mutation data to estimate a cancer-cell fraction (CCF) for each mutation, which were then analyzed to construct phylogenetic trees for clonality analysis to relate the cancer subclones within each patient (Fig. 1a, Supplementary Figure 1A-I).

All paired cases (temporally distinct pre- and post-treatment autopsy samples and spatially distinct metastatic autopsy samples) demonstrated a branched evolution pattern, whereby we detected a common ancestor (harboring truncal alterations), with each sample demonstrating significant subsequent genetic divergence. We noted a striking difference in the truncal status between coding alterations compared to non-coding and structural alterations. Phylogenetic reconstruction demonstrated that somatic exonic mutations, typically in the coding regions of common GBM driver genes, occurred on all branches of the phylogenetic tree, including in isolated subclonal branches (Fig. 1a). However, there were no protein-coding mutations identified as recurrent truncal events across the majority of the cohort. In contrast, despite variable clinical presentations and treatment courses, characteristic recurrent copy-number alterations and the TERT promoter mutation events were nearuniversally present clonally in the pre- and post-treatment autopsy samples (Fig. 1b). Chromosome 10 deletion was clonal in both matched samples in 9 of 9 cases (100\%). Chromosome 7 copy-gain was clonal in the pre- and post-treatment autopsy samples in 8 of 9 cases $(89 \%)$, vs. clonal uniquely in the pre-treatment sample in 1 case (11\%). Chromosome 9p21.3 deletion was clonal in both matched samples in 8 of 9 cases (89\%). TERT promoter mutations were clonal in both pre-treatment and post-treatment autopsy
Table 1. Clinical characteristics of the 12-patient case series

\begin{tabular}{|c|c|}
\hline Variable & Number of Patients \\
\hline Mean Age (yrs) & $62.8 \pm 7.4$ \\
\hline Mean Progression-Free Survival (yrs) & $0.9 \pm 0.8$ \\
\hline Mean Overall Survival (yrs) & $1.4 \pm 1.0$ \\
\hline Female: Male & $4: 8$ \\
\hline \multicolumn{2}{|l|}{ Presenting Symptoms } \\
\hline Headache & $6(50)$ \\
\hline Nausea & $1(8)$ \\
\hline Memory Loss & $3(25)$ \\
\hline Weakness & $5(42)$ \\
\hline Visual Deficit & $2(17)$ \\
\hline Vomiting & $1(8)$ \\
\hline Seizure & $3(25)$ \\
\hline Systemic Metastases & $2(17)$ \\
\hline \multicolumn{2}{|l|}{ Location } \\
\hline Left: Right & 3: 9 \\
\hline Frontal & $4(33)$ \\
\hline Temporal & $6(50)$ \\
\hline Parietal & $2(17)$ \\
\hline \multicolumn{2}{|l|}{ Surgery } \\
\hline Initial Surgery & $12(100)$ \\
\hline Second Surgery & $4(33)$ \\
\hline Third Surgery & $2(17)$ \\
\hline Subtotal Resection & $5(42)$ \\
\hline Gross Total Resection & $6(50)$ \\
\hline Biopsy & $1(8)$ \\
\hline \multicolumn{2}{|l|}{ SNaPshot genotyping } \\
\hline Wildtype & $11(92)$ \\
\hline TP53, 742 C > T (Arg248Trp) & $1(8)$ \\
\hline MGMT methylated: unmethylated & 4: 7 \\
\hline \multicolumn{2}{|l|}{ Therapy } \\
\hline Radiotherapy & $12(100)$ \\
\hline Concurrent temozolomide & $11(92)$ \\
\hline Adjuvant temozolomide & $12(100)$ \\
\hline Mean number of adjuvant temozolomide cycles & $6.3 \pm 4.0$ \\
\hline Surgery at Progression & $2(17)$ \\
\hline \multicolumn{2}{|l|}{ Radiotherapy at Progression } \\
\hline Bevacizumab & $11(92)$ \\
\hline CCNU Salvage Therapy & $4(33)$ \\
\hline \multicolumn{2}{|l|}{ Types of Inhibitors received } \\
\hline EGFR & $4(33)$ \\
\hline HDAC & $1(8)$ \\
\hline MTOR & $2(17)$ \\
\hline MET & $1(8)$ \\
\hline CXCR4 & $1(8)$ \\
\hline VEGF & $1(8)$ \\
\hline
\end{tabular}

Values are presented as the number of patients (\%) unless indicated otherwise. Percentages represent the percentage within a row.

samples in 6 of 7 cases (86\%) and subclonal in the pre-treatment and clonal in the post-treatment sample in 1 case (14\%). In this latter case, the sample had clonal chromosome 10 and 9 p21.3 loss in the pre-treatment and post-treatment autopsy samples, implying that the TERT promoter mutation can occur during gliomagenesis, after chromosome gains and losses. We were 
unable to infer clonality of TERT promoter mutations in the remaining 2 cases due to whole exome sequencing data deriving from a different biopsy sample than the sample used for the Fluidigm assay.
Even though no tumor met the formal criteria for hypermutator phenotype ${ }^{23}$, there was significant genetic divergence in the posttreatment autopsy samples (Fig. 2a-C, Supplementary Figure 1A-I), with higher post-treatment specific mutation rates (single

A

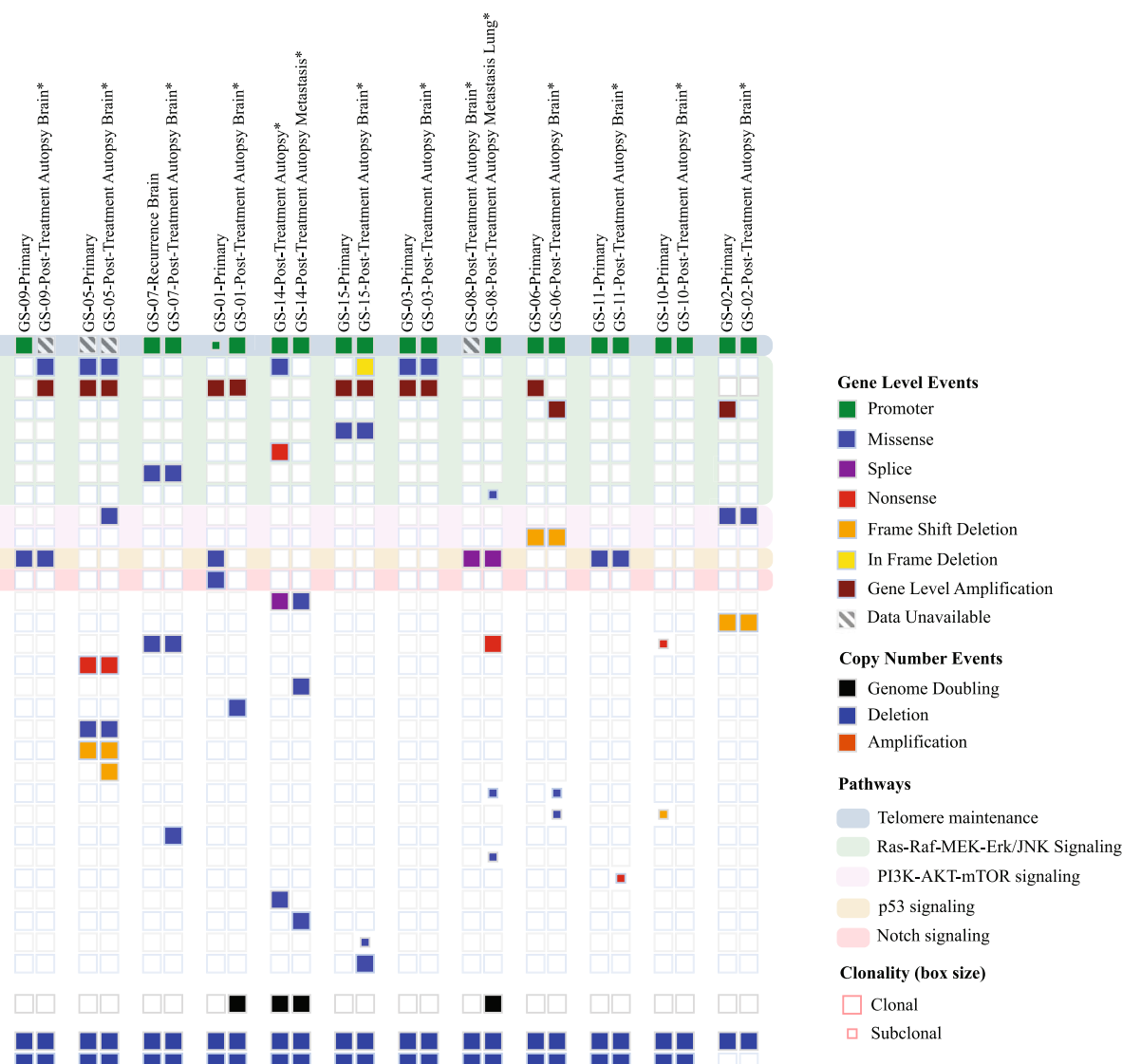

в

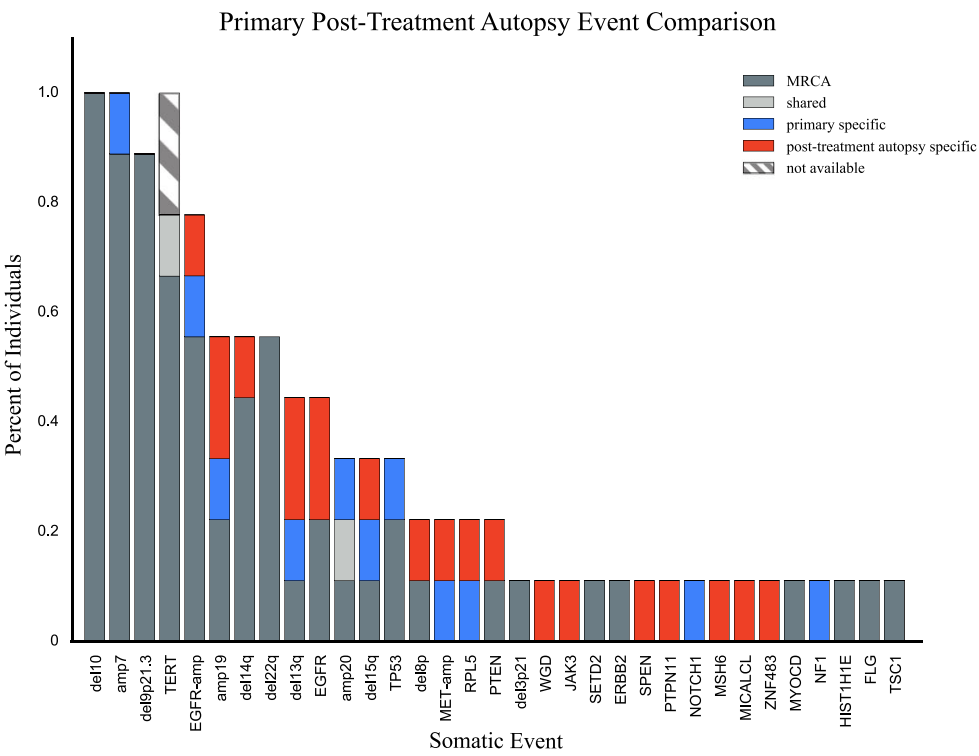


Fig. 1 a. Comut plot of cohort. Columns are grouped together by individual $(n=12)$ in pairs. Both SNVs/indels (top panel) and copy number events (bottom panels) are included. Clonal and subclonal events are demarcated through the size of the box, with empty boxes specifying lack of presence of a mutation in that sample. Genes are grouped together by pathways with high relevance to glioblastoma found on the cBioPortal webpage (http://www.cbioportal.org/). b. Sample-specific bar plot. Only samples with both a pre-treatment primary and posttreatment autopsy sample were included $(n=9)$. Genetic aberrations (SNVs/indels and SCNAs) are represented in each bar, plotted categorically using categories MRCA (Most Recent Common Ancestor - clonal in both samples), shared (present in both samples, at subclonal levels in at least one sample), primary specific (present in primary sample, not present in post-treatment autopsy sample), post-treatment autopsy specific (present in post-treatment autopsy sample, not present in primary sample), not available (data not available - only applies to TERT promoter mutation where Fluidigm assay failed or was unavailable)

a

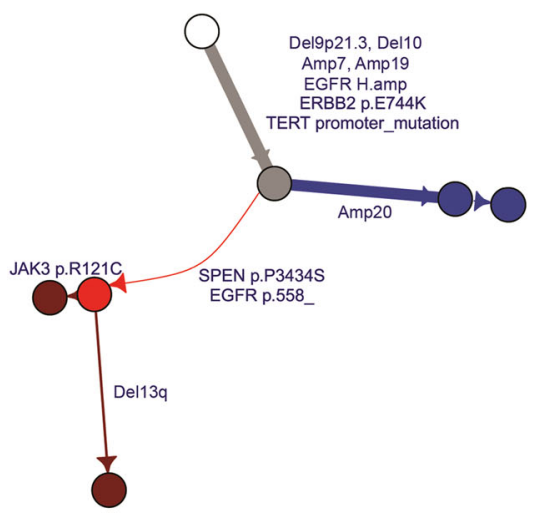

b

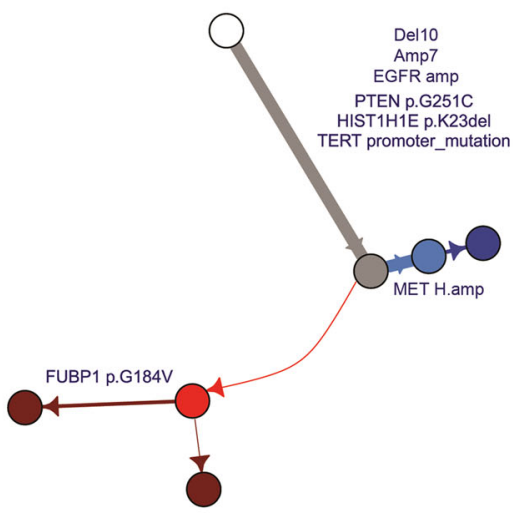

C

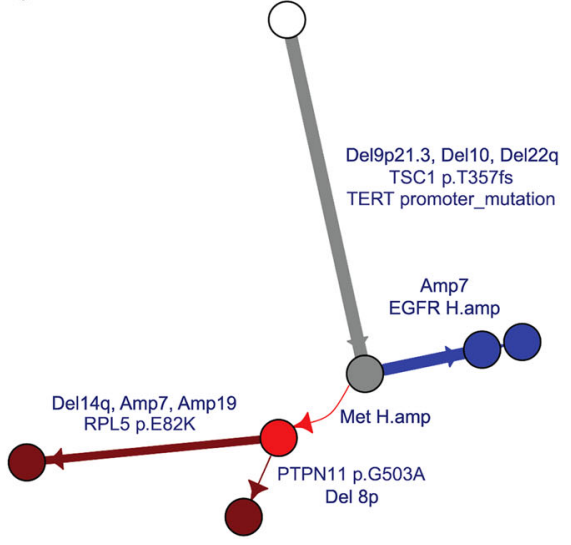

Fig. 2 a-c. Phylogenetic Trees. Phylogenetic trees from representative cases with primary and post-treatment autopsy sample from the same individual. Primary specific clones occur on blue branches, and post-treatment autopsy specific clones occur on red branches, with mutations on driver genes and SCNAs annotated on each branch

nucleotide variants, or SNVs, per Mb and small insertions and deletions, or indels, per $\mathrm{Mb}$ ) compared to the mutation rates respectively in pre-treatment samples (mean 1.202 vs. $0.439 ; p=$ 0.0081, Mann-Whitney test) (Fig. 3, Supplementary Table 1). We could not detect distinct mutational signatures in mutations detected only in the post-treatment autopsy samples, although we did detect significantly more indels in post-treatment autopsy samples than in pre-treatment samples (mean 0.096 vs. $0.015 ; p=$ 0.029, Mann-Whitney test), in addition to the overall higher mutation rate (Fig. 3). Since all patients received radiation as part of their care, we speculate that these are radiation-driven indels. Recognizing the possibility that treatment with temozolomide may in part account for the mutation rates observed in the posttreatment autopsy samples, we examined the overall mutational signature in these samples. The characteristic $\mathrm{CpC}>\mathrm{T}$ temozolomide signature was not detected in any case, suggesting that factors other than exposure to temozolomide contributed to the observed genetic divergence. Detailed evaluation of the mutational signatures specific to pre-treatment and post-treatment autopsy samples uncovered that cytosine to thymidine transitions were predominant in both pre-treatment and post-treatment autopsy cases, which can be attributed to the mutational signature associated with spontaneous deamination of methylated cytosines which occurs naturally and is associated with number of cell divisions ${ }^{28}$, the so-called Signature 1 or "aging" signature (Supplementary Figure 2).

The phylogenetic reconstruction of GBM provided potential insights into possible mechanisms of resistance to different therapies. Below we describe three representative cases that represent a range of treatment regimens. In one case (Fig. 2a: GS15), a patient diagnosed with an IDH wildtype, MGMT promoter methylated, right frontal GBM, underwent a gross total resection, treatment with concurrent temozolomide/radiation, and 12 months of adjuvant temozolomide. 6 months after completion of adjuvant treatment, the patient progressed, was treated with dacomitinib (an oral irreversible tyrosine kinase inhibitor of human epidermal growth factor receptors, including EGFR and ERBB2), experienced clinical and radiographic progression after 2 months of therapy, and ultimately died. The pre-treatment and posttreatment autopsy sample shared a common ancestor with shared deletions (del9p21.3, del10), amplifications (focal EGFR amplification, amp7, amp19) and mutations (ERBB2 E744K, TERT promoter). The pre-treatment sample harbored an additional broad chr20 amplification and the post-treatment autopsy sample had additional alterations, including a clonal six base pair in-frame deletion in EGFR (p.C558_P560delinsS) which has not been previously described. The EGFR indel may represent a resistance mechanism that arose during treatment with the EGFR inhibitor. Evolutionarily, this case illustrates a late phylogenetic event (EGFR indel) altering the dominant treatment-resistant clone, forming a "sequential" narrative of recurrence that is commonly envisioned as the source of treatment resistance.

A second patient (Fig. 2b: GS-02) underwent a resection of a frontal GBM, followed by combined radiation and temozolomide then temozolomide for 6 months and after a clinical and radiographic response, remained stable off of treatment for 6 months, when he was found to have recurrent disease. He was initiated on a clinical trial of a MET inhibitor after his tumor was found to have a c-MET amplification. He progressed after 2 weeks of therapy, and was initiated on bevacizumab and temozolomide with continued progression, and he ultimately died 1 month later. The primary and post-treatment autopsy tumor shared a TERT promoter mutation, copy-loss of chromosome 10, and copy-gain of chromosome 7. Thereafter, phylogenetic parsimony indicates that the pre-treatment and post-treatment samples have a branched "sibling" relationship; neither branch is a subclonal descendent of the other. Notably, the primary tumor had a clonal high-level amplification of MET (with an average of over 70 copies of MET per cancer cell) that was not present in the post-treatment autopsy sample after treatment with the MET inhibitor. Thus, these data suggest that the primary tumor was ineffect spatially heterogeneous, containing a minor reservoir of 


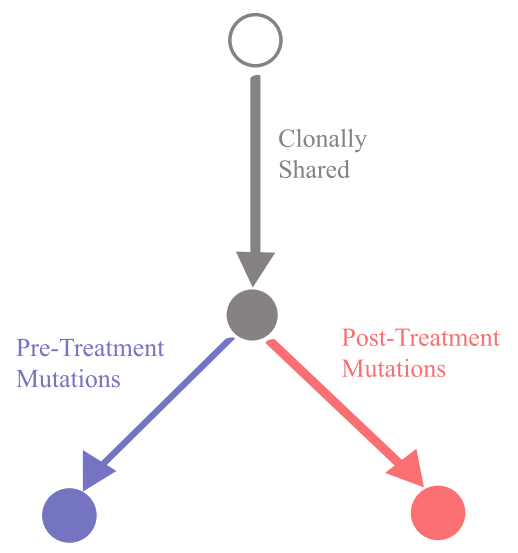

SNV Rate Pre vs. Post-Treatment Autopsy

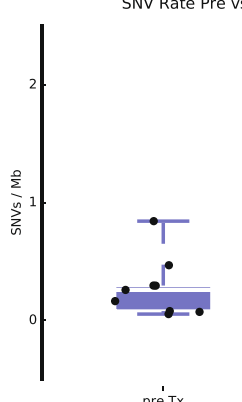

$p=0.0062$

pre Tx

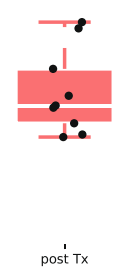

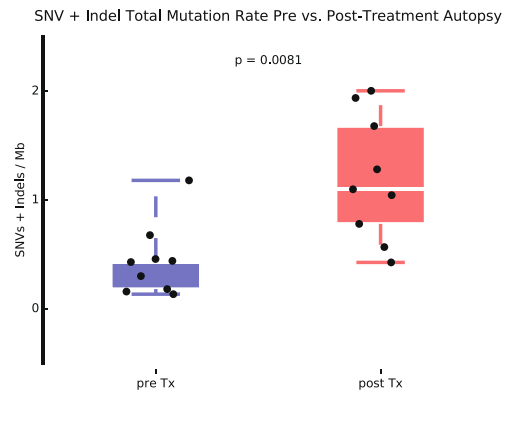
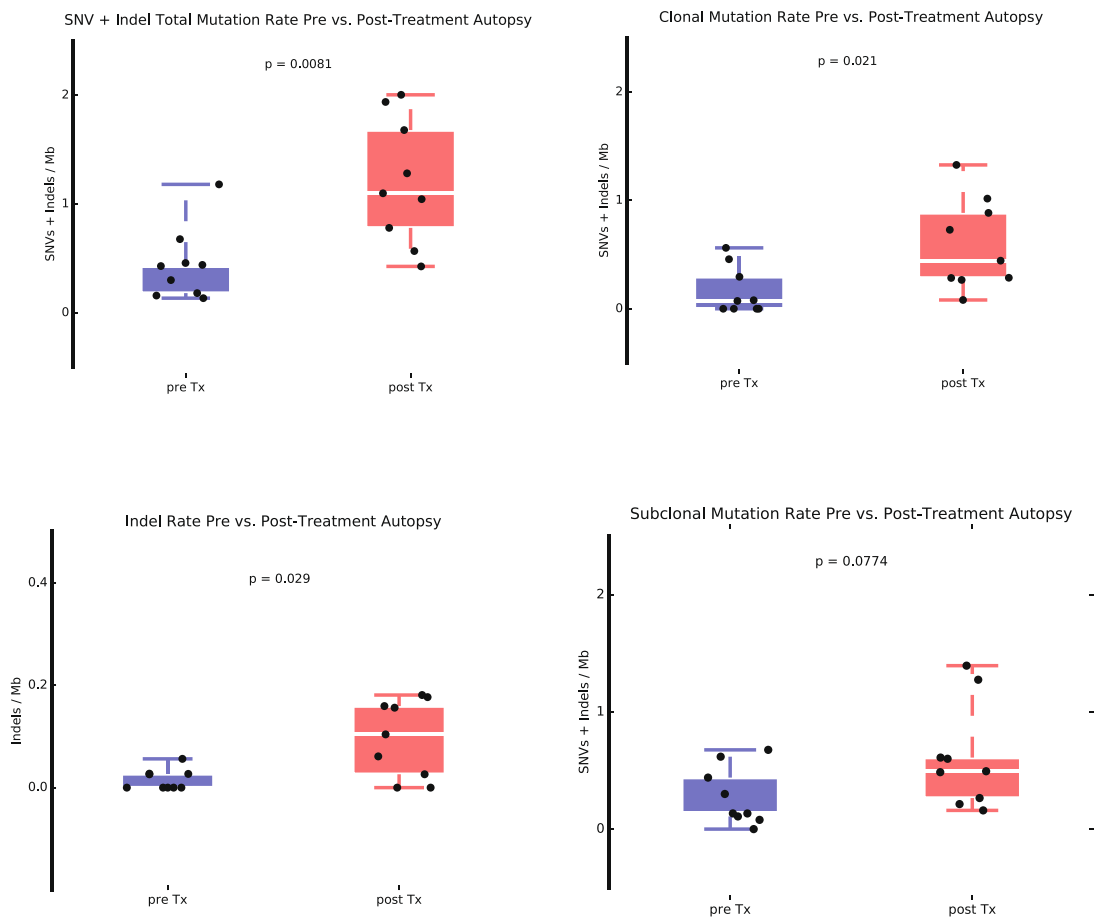

Fig. 3 SNV and indel frequencies per sample $(/ \mathrm{Mb})$ in cases with pre-treatment primary and post-treatment autopsy sample $(n=10)$

TERT mutant, chr10 lost, chr7 gain, MET non-amplified precursor cells that escape targeted therapy.

Similarly, a third patient (Fig. 2c: GS-06) underwent a subtotal resection of a right temporal lobe GBM, followed by adjuvant radiation, temozolomide and an mTOR inhibitor and had progressive disease 7 months later. He was initiated on the VEGF inhibitor tivozanib for 2 months with poor tolerance, and subsequently transitioned to a different VEGF inhibitor (bevacizumab). He progressed after 4 months of bevacizumab monotherapy and died. His pre-treatment and post-treatment autopsy tumors had shared deletions in 9p21.3, 22q, and chromosome 10, amplification of chromosome 7, and a mutation in the TERT promoter. The pre-treatment sample had a high-level focal EGFR amplification, whereas the post-treatment autopsy sample did not have the EGFR amplification and had a number of additional mutations and copy-number alterations including a high-level focal MET amplification. Increased c-Met expression and activity may play a role in resistance during antiangiogenic therapy ${ }^{29-31}$. It is possible that after receiving targeted therapy, episomal EGFR amplifications may have been selected against as a result of the treatment ${ }^{32}$. However, the presence of $17 / 152$ total mutations specific to the primary (vs. 70/152 mutations in the truncal MRCA clone, and $65 / 152$ total mutations specific to the relapse) suggests evolutionarily that the recurrence is not merely a sequential loss of episomal DNA, but rather outgrowth of a related subclone. These latter representative examples (Figs. 2b,c) highlight the late emergence of a highly-divergent clone derived from a shared precursor pre-existing within a treatment-resistant reservoir.

\section{DISCUSSION}

We analyzed the genetic landscape of GBM at the extremes of disease course using our unique biospecimen resource of autopsy specimens. Comparing pre-treatment and autopsy specimens, we demonstrated a common core of four early genetic events (loss of chr10, chr9p21, gain of chr7 and TERT promoter mutations), occurring before the divergence of primary tumor and posttreatment tumor, which are detectable in virtually every case.

More generally, our results suggest that there is significant inter-patient heterogeneity with respect to protein coding (exonic) mutations and that the key early events in GBM phylogeny are not mutations in exonic regions. Exonic mutations in genes such as TP53 and EGFR, although well-established and thought of as key clinically-actionable mutations leading to recurrence and progression, were found in our cohort primarily as later events with respect to GBM phylogeny, as evidenced by their presence on both branches of the evolutionary tree and absence on its trunk. A number of oncogenic alterations emerged after treatment with targeted therapies, indicative of ongoing genomic evolution.

In contrast, we show that TERT promoter mutations are present in nearly all cases, from pre-treatment and at the time of death. Interestingly, in a single case the TERT promoter mutation was initially subclonal and later became clonal, whereas the copy gains and losses were clonal throughout, suggesting that the TERT promoter mutation did not precede the copy number alterations, and was therefore not a requirement for copy gains and losses. Indeed, unlike other brain tumors such as meningomas ${ }^{33}$ where copy number variations arise late during disease progression, our data suggest that copy number changes are amongst the earliest drivers of gliomagenesis.

Collectively, these core four alterations define the shared-origin cell population from which later-emerging recurrences arise in the majority of patients with GBM. Further studies may identify additional truncal alterations contained within the shared-cell precursor. Nevertheless, these alterations point towards the significance of non-coding and structural alterations in gliomagenesis, with significant implications for treatment strategy. Therapies that target protein coding mutations have efficacy in other cancers but lack durable activity in GBM. ${ }^{34-36}$ Our findings indicate this may be because these therapies do not target the 
entire cellular reservoir, primarily characterized by non-coding and structural changes. Comprehensive analysis of the functional roles of these early events and development of novel therapeutic strategies to target them should be given priority.

\section{METHODS}

We identified 12 GBM cases with temporally $(n=10)$ and spatially $(n=2)$ distinct tumor tissue. The study was conducted in accordance with the Declaration of Helsinki. The study was reviewed and approved by the human subjects institutional review board of the Dana-Farber Cancer/ Harvard Cancer Center. All patients provided written informed consent for genetic analysis. A board-certified neuropathologist (M.F.) confirmed the histologic diagnoses and selected representative formalin fixed paraffin embedded samples that had an estimated purity of greater than or equal to $40 \%$.

Sequence data generation and pre-processing

Whole exome sequencing was performed using the sequencing platforms at the Broad Institute. Details of whole exome library construction have been previously described. ${ }^{37}$

A binary SAM file (BAM) file was generated for each sample using the sequencing data processing pipeline known as "Picard" (http:// broadinstitute.github.io/picard/). Picard consists of four previously described $^{38}$ steps, detailed below.

(1) Alignment to the genome. Alignment was performed using $\mathrm{BWA}^{39}$ (http://bio-bwa.sourceforge.net/) to the NCBI Human Reference Genome GRCh37/hg19. The reads in the BAM file are sorted according to their chromosomal position. Unaligned reads are also stored in the BAM file such that all reads that passed the Illumina quality filter (PF reads) are kept in the BAM.

(2) Base-quality recalibration. Each base is associated with a Phred-like quality $\mathrm{Q}$ score representing the probability that the base call is erroneous. The Q score represent $-10^{*} \log$ (probability of error), rounded to an integer value. In order to make sure that Q30 bases indeed have a 1 in a 1000 chance of being wrong we used a GATK tool (http://www.broadinstitute. org/gatk) that empirically recalibrates the qualities based on the original $Q$ score (generated by the Illumina software), the read-cycle, the lane, the tile, the base in question and the preceding base. The original quality scores are also kept in the BAM file in the read-level OQ tag.

(3) Aggregation of lane and library-level data. Multiple lanes and libraries are aggregated into a single BAM per sample. Lane-level BAM files are combined to library-level BAM files that are then combined to sample-level BAM files. The BAM files contain read-groups that represent the library and lane information. Information regarding the read groups appears in the BAM header (see the BAM file specifications in http://samtools.sourceforge. net/SAM1.pdf).

(4) Marking of duplicated reads. Molecular duplicates are flagged using the MarkDuplicates algorithm from Picard (http://broadinstitute.github.io/ picard/). The method identifies pairs of reads in which both ends map to the exact same genomic position as being multiple reads of the same DNA molecule and hence marks all but the first as duplicates.

Targeted sequencing of TERT promoter region. Targeted sequencing of the TERT promoter region was also performed for each tumor sample using Fluidigm sequencing technologies. A portion of the TERT promoter region [273 bp; Chr5: 1,295,040-1,295,313 (hg19)] was amplified and sequenced in 20 samples. These PCRs were carried out in two reactions. Round-1 PCR primers contained target-specific sequences and Illumina adapter sequences, producing a product of $341 \mathrm{bp}$. Round-2 PCR was a "tailing" PCR in that PCR2 primers contained overlap of the Illumina adapter sequence, as well as flow cell attachment sequence, and an eight bp index on the reverse primer between the adapter sequence and flow cell attachment sequence. This tailing PCR produced sequence-ready constructs of $398 \mathrm{bp}$ that did not require further library construction. Firstround PCR was carried out using the Platinum Pfx DNA polymerase kit (Life Technologies, Inc.). PCR1 reactions consisted of $50 \mathrm{ul}: 2 \mathrm{ul} \mathrm{DNA} \mathrm{(at} \sim 25 \mathrm{ng} / \mathrm{ul}$ ), $3 \mathrm{ul} \mathrm{mixed} \mathrm{F/R} \mathrm{tailed} \mathrm{target-specific} \mathrm{primer} \mathrm{(at} 20 \mathrm{uM}$ mixed), 5 ul 10X Pfx amplification buffer, $1.5 \mathrm{ul}$ dNTPs [at $10 \mathrm{mM}$ each (Agilent Technologies)],
$0.8 \mathrm{ul} \mathrm{Pfx}$ Platinum DNA polymerase, $1 \mathrm{ul} \mathrm{MgSO}_{4}$ (at $50 \mathrm{mM}$ ), $5 \mathrm{ul} 10 \mathrm{X} \mathrm{Pfx}$ Enhancer Solution, and $31.7 \mathrm{ul}$ nuclease free water. The polymerase $(0.4 \mathrm{ul}$ polymerase $+1.6 \mathrm{ul}$ water) was added to reactions after $1 \mathrm{~min}$ at $95^{\circ} \mathrm{C}$. Thermal cycling consisted of $95^{\circ} \mathrm{C}$ for $5 \mathrm{~min}$ (paused at $1 \mathrm{~min}$ to add polymerase), 30 cycles of $\left[95^{\circ} \mathrm{C} 30 \mathrm{sec}, 55^{\circ} \mathrm{C} 30 \mathrm{sec}, 68^{\circ} \mathrm{C} 1 \mathrm{~min}\right]$. A sampling of PCR1 products (and negative control) were visually inspected on the Lab Chip GX II Caliper Instrument (Perkin Elmer). Next, secondround index-tailing PCRs were carried out using the HiFi Library Amplification kit (Kapa Biosystems, Inc.). PCR2 reactions consisted of 60 ul: 10 ul PCR1 product, 12 ul 5X Kapa HiFi Fidelity Buffer, 1 ul dNTPs (25 uM), 1 ul Kapa Hifi HotStart Enzyme, 32 ul nuclease free water, and 4 ul PCR2 F/R index-primer mix ( $25 \mathrm{uM}$ mixed, plate of 96). Thermal cycling consisted of $98^{\circ} \mathrm{C}$ for $45 \mathrm{sec}, 8 \mathrm{cycles}$ of $\left[98^{\circ} \mathrm{C} 15 \mathrm{sec}, 60^{\circ} \mathrm{C} 30 \mathrm{sec}, 72^{\circ} \mathrm{C} 30\right.$ sec] and $1 \mathrm{~min}$ at $72^{\circ} \mathrm{C}$. Indexed amplicons were pooled in equal volumes (96 reactions per pool), and purified using 1.5X solid-phase reversible immobilization (SPRI) cleanup with Agencourt Ampure XP beads (Beckman Coulter). Final amplicon library pools were visually inspected and quantified on a BioAnalyzer (Agilent Technologies). The library was requantified by SYBR green QPCR before denaturing and cluster generation. PhiX library, derived from the well-characterized and small PhiX genome, was spiked in at $15 \%$ to add diversity to single-amplicon clusters for improved cluster imaging. One MiSeq run $(2 \times 150 \mathrm{bp}$ paired end with standard sequencing primers) was carried out for each pool of indexed amplicons, using standard sequencing protocols (Illumina).

\section{Cancer genome analysis pipeline}

Whole exome sequencing data was analyzed using Firehose (developed at the Broad Institute; https://www.broadinstitute.org/cancer/cga). All tumornormal pairs passed the Firehose QC pipeline, which included testing for DNA contamination of a sample from other individuals using the Contest algorithm, ${ }^{40}$ as well as cross-checking lane fingerprints. A more detailed description of the QC pipeline can be found here. ${ }^{38}$

Identification of somatic single nucleotide variants (SSNVs) and small insertions and deletions (indels). Candidate SSNVs were detected using the point mutation calling algorithm MuTect, ${ }^{41}$ ran on each tumor-normal pair. All mutations were filtered using the oxoG filter, which filters mutations that arise due to oxidation of a $\mathrm{G}$ base pair on only one strand during fragmentation. ${ }^{42}$ Since the tumor samples analyzed for this study were formalin-fixed paraffin-embedded (FFPE) samples, candidate SSNVs were then filtered using a Panel of Normals (PoN) filter, comprised of 374 FFPE normal samples. This step was taken to remove potential sequencing artifacts and potential germline sites missed in the matched normal sample. Mutant and reference allele counts were also estimated at known hotspot mutation sites in the TERT promoter region (p.C228T and p.C250T) in the Fluidigm targeted sequencing BAM files. Candidate indels were detected using the Strelka indel calling algorithm ${ }^{43}$ on each tumor-normal pair. Similarly to SSNV filtering, indel calls were filtered using the same PoN filter. While many artifactual mutations were removed in the various filtering processes, we still manually reviewed all validated mutations to remove further artifacts, which included mutations called on low mapping quality reads, mutations called on reads which also contained indels and other low allelic fraction point mutations, mutation supported only by duplicate reads, mutations with strong orientation bias, as well as mutations called in poorly mapping regions. In total, 141 mutations (3 indels, 138 SSNVs) were manually filtered across 1,476 mutations calls from all 12 patients (filter rate of $9.3 \%$ ).

Identification of somatic copy number alterations (SCNAs). A coverage profile for each tumor sample was estimated using the ReCapSeg tool (http://gatkforums.broadinstitute.org/gatk/discussion/5640/recapsegoverview). This tool works by first normalizing read coverage over each target segment with the total number of aligned reads. Next, coverage at every segment is normalized against the coverage across a Panel of Normals (PoN) generated from 25 normal FFPE samples sequenced using the same target regions. Next, target regions are merged to form segments corresponding to the same copy number event using the circular binary segmentation algorithm. ${ }^{44}$ Allelic copy ratio was then estimated by measuring allelic fraction of germline heterozygous SNPs in each tumor sample (found in matched normal samples), and combining these estimates with the observed copy ratio of each segment using the AllelicCapseg tool (http://archive.broadinstitute.org/cancer/cga/acsbeta). Finally, somatic copy number alterations were estimated by running the ABSOLUTE algorithm, ${ }^{12}$ which maps allelic copy ratios to allelic copy 
numbers via a linear transformation after correcting for purity and ploidy of the sample.

Calculation of cancer cell fractions (CCFS) of SSNVs and indels, and subsequent phylogenetic analysis. The CCF distribution of each point mutation (both SSNVs and indels) was estimated using ABSOLUTE. Point mutations were force-called across each tumor sample belonging to each patient; a process in which the aggregate set of all point mutations found in each tumor sample belonging to a patient was formed, and the mutant and reference allele counts in each sample estimated using samtools (http://samtools.sourceforge.net/). Reads were only included if they had a unique pair, had mapping quality greater than or equal to 5 , and a base quality at the site of interest greater than or equal to 20 . The mutant and reference allele counts for each mutation in the force-called set of mutations was used as input to ABSOLUTE, which estimates the CCF distribution of each point mutation based on purity and local ploidy of the site.

Mutation CCFs were subsequently clustered across each individual using a Bayesian clustering method. The final clusters were found by sampling from a Dirichlet process using a Markov chain Monte Carlo (MCMC) sampler, as described here. ${ }^{24,45}$ Five hundred MCMC iterations were used to find the final number of clusters. Phylogenetic trees were then drawn for each patient based on the CCF estimates of these clusters.

While our resolution to detect mutations at low cancer cell fraction is limited, we can still estimate our power to detect mutations at a CCF of 0.05 given the tumor's purity and local absolute copy number, calculated as follows. For a mutation with coverage $C$ (total number of reads mapping to locus of mutation) in a sample with purity $\mathrm{P}$, and total number of allelic copies in the region containing the mutation, $\mathrm{N}$, we calculate the expected allele fraction of mutation with CCF of 0.05 as:

$a f=\frac{0.05 * P}{2 *(1-P)+N * P}$

The power to detect a mutation at CCF of 0.05 at that locus is then:

$\sum_{i=1}^{C} \operatorname{Pr}(i ; C$, af $)$, where $\operatorname{Pr}(k ; n, p)$ is the binomial p.m.f.

We found that we had greater than $50 \%$ power to detect mutations at CCF of 0.05 in 5 point mutations in driver genes which were found in a clone not present in the primary but clonal in the metastasis (MSH6_p. F11Y in PKB-GS-001, PTEN p.I33T in PKB-GS-005, EGFR_p.A289V in PKB-GS009, SPEN_p.P3434S in PKB-GS-015, and EGFR_p.558_ in PKB-GS-015), while 2 point mutations in driver genes had power to detect less than $50 \%$ in similar clones (EGFR_p.C636F in PKB-GS-005 and MICALCL_p.G245fs in PKB-GS-005). Although the MSH6 p.F11Y had one supporting read in the primary sample in PKB-GS-001, it clustered with a clone with estimated CCF of 0.01 , below our threshold of subclonal presence of a clone.

\section{Data availability statement}

Supplementary Table 2 includes the mutation annotation format (MAF) file for all patients sequenced. Sequence data that support the findings of this study have been deposited in dbGaP with the accession code phs1424.v1.p1.

\section{ACKNOWLEDGEMENTS}

G.G. was partially funded by the Paul C. Zamecnik, M.D., Chair in Oncology at Massachusetts General Hospital. P.K.B. was partially funded from ABTA, Brain Science Foundation, Damon Runyon Cancer Research Foundation and Susan G. Komen Foundation.

\section{AUTHOR CONTRIBUTIONS}

P.K.B. and T.T.B. designed the study. C.M.G., N.N., M.S.B., M.D.A., K.H., E.A. and A.K. coordinated tissue acquisition, processed tissue samples, and performed DNA extractions. C.M.G., U.N.C., and M.B. collected clinical information. A.C., S.P., T.T.B., and E.R.G. consented patients for this study. M.P.F. performed pathology review. P.K.B., N. N., and D.P.C. wrote the manuscript. N.N., D.R., I.L., D.L. and G.G. performed computational analyses. P.K.B. supervised the sequencing. C.M.G. identified patients from the tissue bank. P.K.B., D.P.C., G.G. and T.T.B. supervised the study. M.L.S. and A.C. contributed to the interpretation of the results and manuscript writing. All authors discussed the results and implications and edited the manuscript.

\section{ADDITIONAL INFORMATION}

Supplementary Information accompanies the paper on the npj Precision Oncology website (doi:10.1038/s41698-017-0035-9).

Competing interests: Dr. Batchelor has consulted for Merck, NXDC and Amgen. Dr. Brastianos has consulted for Merck, Genentech-Roche, Angiochem and Lilly.

Publisher's note: Springer Nature remains neutral with regard to jurisdictional claims in published maps and institutional affiliations.

\section{REFERENCES}

1. Brennan, C. W. et al. The somatic genomic landscape of glioblastoma. Cell 155, 462-477 (2013)

2. Frattini, V. et al. The integrated landscape of driver genomic alterations in glioblastoma. Nat Genet 45, 1141-1149 (2013).

3. Parsons, D. W. et al. An integrated genomic analysis of human glioblastoma multiforme. Science 321, 1807-1812 (2008).

4. Nowell, P. C. The clonal evolution of tumor cell populations. Science 194, 23-28 (1976).

5. Sidransky, D. et al. Clonal expansion of p53 mutant cells is associated with brain tumour progression. Nature 355, 846-847 (1992).

6. Anderson, K. et al. Genetic variegation of clonal architecture and propagating cells in leukaemia. Nature 469, 356-361 (2011).

7. Yap, T. A., Gerlinger, M., Futreal, P. A., Pusztai, L. \& Swanton, C. Intratumor heterogeneity: seeing the wood for the trees. Sci Transl Med 4, 127ps10 (2012).

8. Campbell, P. J. et al. The patterns and dynamics of genomic instability in metastatic pancreatic cancer. Nature 467, 1109-1113 (2010).

9. Gerlinger, M. et al. Intratumor heterogeneity and branched evolution revealed by multiregion sequencing. N Engl J Med 366, 883-892 (2012).

10. Liu, W. et al. Copy number analysis indicates monoclonal origin of lethal metastatic prostate cancer. Nat Med 15, 559-565 (2009).

11. Navin, N. et al. Tumour evolution inferred by single-cell sequencing. Nature $\mathbf{4 7 2}$ 90-94 (2011)

12. Carter, S.L. et al. Absolute quantification of somatic DNA alterations in human cancer. Nature biotechnology 30, 413-421 (2012).

13. Ding, L. et al. Genome remodelling in a basal-like breast cancer metastasis and xenograft. Nature 464, 999-1005 (2010).

14. Nik-Zainal, S. et al. Mutational processes molding the genomes of 21 breast cancers. Cell 149, 979-993 (2012).

15. Shah, S. P. et al. Mutational evolution in a lobular breast tumour profiled at single nucleotide resolution. Nature 461, 809-813 (2009).

16. Lawrence, M. S. et al. Mutational heterogeneity in cancer and the search for new cancer-associated genes. Nature 499, 214-218 (2013).

17. Gill, B. J. et al. MRI-localized biopsies reveal subtype-specific differences in molecular and cellular composition at the margins of glioblastoma. Proc Natl Acad Sci U S A 111, 12550-12555 (2014).

18. Snuderl, M. et al. Mosaic amplification of multiple receptor tyrosine kinase genes in glioblastoma. Cancer Cell 20, 810-817 (2011).

19. Sottoriva, A. et al. Intratumor heterogeneity in human glioblastoma reflects cancer evolutionary dynamics. Proc Natl Acad Sci U S A 110, 4009-4014 (2013).

20. Wang, J. et al. Clonal evolution of glioblastoma under therapy. Nat Genet $\mathbf{4 8}$, 768-776 (2016).

21. Kim, H. et al. Whole-genome and multisector exome sequencing of primary and post-treatment glioblastoma reveals patterns of tumor evolution. Genome Res $\mathbf{2 5}$ 316-327 (2015)

22. Kim, J. et al. Spatiotemporal evolution of the primary glioblastoma genome. Cancer Cell 28, 318-328 (2015).

23. Hunter, C. et al. A hypermutation phenotype and somatic MSH6 mutations in recurrent human malignant gliomas after alkylator chemotherapy. Cancer Res $\mathbf{6 6}$ 3987-3991 (2006).

24. Brastianos, P. K. et al. Genomic characterization of brain metastases reveals branched evolution and potential therapeutic targets. Cancer Discov 5 1164-1177 (2015).

25. Landau, D.A., Carter, S.L., Getz, G. \& Wu, C.J. Clonal evolution in hematological malignancies and therapeutic implications. Leukemia 28, 34-43 (2013).

26. Landau, D. A. et al. Evolution and impact of subclonal mutations in chronic lymphocytic leukemia. Cell 152, 714-726 (2013).

27. McFadden, D. G. et al. Genetic and clonal dissection of murine small cell lung carcinoma progression by genome sequencing. Cell 156, 1298-1311 (2014).

28. Alexandrov, L. B. et al. Signatures of mutational processes in human cancer. Nature 500, 415-421 (2013).

29. Kong, D. S. et al. Prognostic significance of c-Met expression in glioblastomas. Cancer 115, 140-148 (2009). 
30. Abounader, R. \& Laterra, J. Scatter factor/hepatocyte growth factor in brain tumor growth and angiogenesis. Neuro Oncol 7, 436-451 (2005).

31. Laterra, J. et al. Scatter factor/hepatocyte growth factor gene transfer enhances glioma growth and angiogenesis in vivo. Lab Invest 76, 565-577 (1997).

32. Nathanson, D. A. et al. Targeted therapy resistance mediated by dynamic regulation of extrachromosomal mutant EGFR DNA. Science 343, 72-76 (2014).

33. Brastianos, P. K. et al. Genomic sequencing of meningiomas identifies oncogenic SMO and AKT1 mutations. Nat Genet 45, 285-289 (2013).

34. Chakravarti, A. et al. RTOG 0211: a phase $1 / 2$ study of radiation therapy with concurrent gefitinib for newly diagnosed glioblastoma patients. Int J Radiat Oncol Biol Phys 85, 1206-1211 (2013).

35. Ma, D. J. et al. A phase II trial of everolimus, temozolomide, and radiotherapy in patients with newly diagnosed glioblastoma: NCCTG N057K. Neuro Oncol 17, 1261-1269 (2015)

36. Raizer, J. J. et al. A phase II study of bevacizumab and erlotinib after radiation and temozolomide in MGMT unmethylated GBM patients. J Neurooncol 126, 185-192 (2016).

37. Berger, M. F. et al. The genomic complexity of primary human prostate cancer. Nature 470, 214-220 (2011).

38. Chapman, M. A. et al. Initial genome sequencing and analysis of multiple myeloma. Nature 471, 467-472 (2011).

39. Li, H. \& Durbin, R. Fast and accurate long-read alignment with Burrows-Wheeler transform. Bioinformatics 26, 589-595 (2010).

40. Cibulskis, K. et al. ContEst: estimating cross-contamination of human samples in next-generation sequencing data. Bioinformatics 27, 2601-2602 (2011).

41. Cibulskis, K. et al. Sensitive detection of somatic point mutations in impure and heterogeneous cancer samples. Nat Biotechnol 31, 213-219 (2013).
42. Costello, M. et al. Discovery and characterization of artifactual mutations in deep coverage targeted capture sequencing data due to oxidative DNA damage during sample preparation. Nucleic Acids Res. 41, e67 (2013).

43. Saunders, C. T. et al. Strelka: accurate somatic small-variant calling from sequenced tumor-normal sample pairs. Bioinformatics 28, 1811-1817 (2012).

44. Olshen, A. B., Venkatraman, E. S., Lucito, R. \& Wigler, M. Circular binary segmentation for the analysis of array-based DNA copy number data. Biostatistics $\mathbf{5}$, 557-572 (2004)

45. Stachler, M. D. et al. Paired exome analysis of Barrett's esophagus and adenocarcinoma. Nat Genet 47, 1047-1055 (2015).

\section{(c) (†)}

Open Access This article is licensed under a Creative Commons Attribution 4.0 International License, which permits use, sharing, adaptation, distribution and reproduction in any medium or format, as long as you give appropriate credit to the original author(s) and the source, provide a link to the Creative Commons license, and indicate if changes were made. The images or other third party material in this article are included in the article's Creative Commons license, unless indicated otherwise in a credit line to the material. If material is not included in the article's Creative Commons license and your intended use is not permitted by statutory regulation or exceeds the permitted use, you will need to obtain permission directly from the copyright holder. To view a copy of this license, visit http://creativecommons. org/licenses/by/4.0/.

(c) The Author(s) 2017 